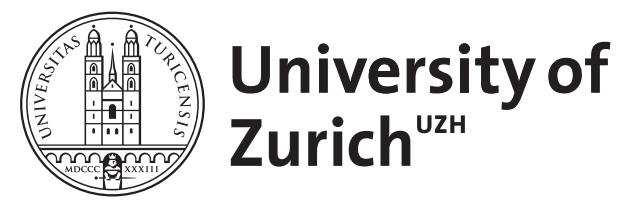

\title{
Breastfed at Tiffany's
}

Hennet, Thierry ; Borsig, Lubor

\begin{abstract}
The importance of breast milk for the growing infant is undisputed; breastfeeding decreases infantile mortality by tenfold and decreases the incidence of infectious diseases. Despite its recognized benefits, the structural richness of breast milk has also impeded the characterization of the multiple effects of milk components on infant physiology. However, the important roles of some components of breast milk are beginning to be dissected. For instance, molecules such as immunoglobulin A (IgA) and milk oligosaccharides protect from gastrointestinal infections and influence the development of the gut microbiota. Deciphering the complex composition of breast milk brings to light multifaceted contributions that combine to make breast milk the ultimate personalized medicine.
\end{abstract}

DOI: https://doi.org/10.1016/j.tibs.2016.02.008

Posted at the Zurich Open Repository and Archive, University of Zurich

ZORA URL: https://doi.org/10.5167/uzh-128079

Journal Article

Accepted Version

Originally published at:

Hennet, Thierry; Borsig, Lubor (2016). Breastfed at Tiffany's. Trends in Biochemical Sciences, 41(6):508518.

DOI: https://doi.org/10.1016/j.tibs.2016.02.008 


\section{Breastfed at Tiffany's}

Thierry Hennet, Lubor Borsig

3 Department of Physiology and Zurich Center for Integrative Human Physiology, University of

4 Zürich, Winterthurerstrasse 190, Zürich, Switzerland

5

6 Corresponding author: Thierry Hennet (thierry.hennet@uzh.ch)

7

8 Keywords

9 Breast milk, microbiota, IgA, lactose, lactase, oligosaccharide

11 Abstract

12 The importance of breast milk for the growing infant is undisputed; breastfeeding decreases

13 infantile mortality by 10 -fold and decreases the incidence of infectious diseases. Despite its

14 recognized benefits, the structural richness of breast milk has also impeded the

15 characterization of the multiple roles of milk components on infant physiology. However,

16 the important roles of some components of breast milk are beginning to be dissected. For

17 instance, molecules such as immunoglobulin A and milk oligosaccharides protect from

18 gastrointestinal infections and influence the development of the gut microbiota. Deciphering

19 the complex composition of breast milk brings to light multifaceted contributions that sum

20 up to make breast milk the ultimate personalized medicine. 


\section{Breastfeeding protects child and mother}

22

24

Breast milk is often described as being the gold standard of infant nutrition, because breast milk provides all macronutrients and vitamins required for the optimal development of the suckling infant. Recent meta-analyses underline the beneficial effects of breastfeeding on short-term child health by decreasing infant mortality and morbidity [1], and on long-term development by reducing the risk for obesity [2]. Health benefits also extend to the nursing mother, as breastfeeding protects against breast cancer [3]. Considering the strong impact of breast milk on child health, the WHO recommends exclusive breastfeeding for the first six months of life and breast milk as a complement to solid food for at least an additional year [4].

More than just a gold standard, breast milk is a jewel shaped by millions of years of evolution that chiseled a perfect multi-functional fluid. In fact, beyond the supply of nutrients and vitamins, breast milk provides bioactive factors including immunoglobulins, cytokines, antimicrobial proteins, hormones, and oligosaccharides, which work in concert to fortify mucosal immunity, shape the gut microbiota, stimulate body growth, and even to regulate birth spacing in mothers. Breast milk is a rich fluid that fulfills multiple tasks as discussed in this Review.

\section{Breast milk is a meal}

Let us begin by crunching some numbers. At the beginning of lactation, each human breast produces on average $450 \mathrm{~g}$ of milk daily. After 15 months, the daily output still reaches up to $200 \mathrm{~g}$ of milk, although the amount largely depends on the intensity of breastfeeding [5]. To accommodate this increased energy expenditure the nursing mother has to increase her daily caloric intake of about $2000 \mathrm{kcal}$ [6] by taking up an additional $500 \mathrm{kcal}$. This 
supplement nearly compensates for the $625 \mathrm{kcal}$ required for the daily production of 700 to $900 \mathrm{~g}$ of breast milk. The process itself is very efficient as the conversion of dietary energy to milk energy has been estimated to reach $80 \%$ [7]. Altogether, the energy expenditure bound to milk production is considerable and is comparable to the daily caloric uptake of the brain $[8]$.

The true structural and functional richness of breast milk emanates from the multitude of components included in the fat, protein, and carbohydrate fractions. The composition of breast milk differs largely between mammals. For example, marine mammals have a milk rich in fat, fast-growing mammals have a milk rich in proteins, marsupials and primates have a milk rich in carbohydrates [9]. In humans, the ethnicity and age of nursing mothers have little impact on the overall milk composition [10], but the stage of lactation has by far the largest effect on the individual classes of macronutrient. In general, colostrum (see Glossary) has high concentrations of bioactive proteins and oligosaccharides, whereas mature milk has proportionally high levels of lipids and caseins. The maternal diet has little effect on most macronutrient classes, although dietary lipids definitively influence the fatty acid composition of breast milk [11]. Lipids are the largest source of calories, yielding $40-50 \%$ of the total dietary energy of breast milk [12]. In addition to triglycerides and cholesterol, the lipid fraction of early milk includes several lipid mediators, such as anti-inflammatory lipoxins and resolvins [13]. Milk proteins are often subdivided into insoluble caseins that build micelles, and soluble whey proteins, which include bioactive proteins such as secreted immunoglobulin A ( $\operatorname{sg} A)$, lactoferrin, lysozyme, and $\alpha$-lactalbumin. The carbohydrate fraction consists of lactose (50-70 g/l) and complex oligosaccharides (5-10 g/l). Despite its structural simplicity and the universal occurrence of glucose (Glc) and galactose (Gal) in 
67 living organisms, the disaccharide lactose that combines Glc and Gal is only found in

68 mammals.

\section{Breast milk is a clock}

Lactose is synthesized in the secretory epithelium of the mammary gland by coupling Gal in a $\beta 1-4$ linkage to Glc. Lactose synthase (EC 2.4.1.22) is a dimer comprising the $\beta 1-4$ galactosyltransferase B4GALT1 [14] found in the Golgi apparatus of all cells and $\alpha$ lactalbumin [15], which is specifically expressed in the mammary gland. In the absence of $\alpha$ lactalbumin, B4GALT1 has a low affinity for Glc as acceptor substrate and preferentially transfers Gal to N-acetylglucosamine (GlcNAc). While associated with $\alpha$-lactalbumin, the affinity of B4GALT1 for Glc increases by 1000-fold [16], thereby enabling the formation of lactose. During pregnancy the expression of $\alpha$-lactalbumin is inhibited by high levels of circulating progesterone [17] that counteracts the stimulatory effect of the pituitary hormone prolactin, the levels of which rise strongly in the second half of gestation. At parturition, progesterone drops while sustained prolactin secretion induces $\alpha$-lactalbumin expression, hence stimulating milk production. In addition to its role as nutrient, lactose is also used as an acceptor substrate for the synthesis of a multitude of oligosaccharides, which will be addressed in the next section.

After ingestion of breast milk, lactose must be cleaved back to Gal and Glc in order to be absorbed and used as a source of energy by the suckling infant. The enzyme responsible for lactose cleavage is the $\beta$-galactosidase lactase [18], which is expressed at the brush border membrane of the small intestine. Lactase expression is tightly regulated and is progressively turned off in the majority of children around two to three years of age (Fig. 1). Decreased lactase activity leads to the passage of lactose to the large intestine, where it is metabolized 
by microbes, thereby releasing hydrogen, methane, carbon dioxide, and lactate [19]. These

91 fermentation products cause bloating, abdominal cramps, and nausea, which are the typical symptoms of lactose intolerance. The emergence of such symptoms will lead the nursed child to reject breast milk and eventually to natural weaning.

Ovarian follicle maturation is suppressed during lactation because of the elevated prolactin and low gonadotropin levels in nursing mothers [20]. This phenomenon prevents a new pregnancy when a mother dedicates a major fraction of her energy expenditure to breastfeeding. Accordingly, lactase repression and the transition to a state of lactose intolerance can be seen as a natural clock regulating weaning and thereby the return to fertility for the mother (Fig. 1). Therefore, the lactose-lactase system has been suggested to

100 act as a biological timer controlling birth spacing in humans [21].

101 Whereas the majority of mankind loses lactase expression during early childhood, about $40 \%$ of the human population shows lifelong lactase persistence. The geographical distribution of 103 lactase persistence is striking as it is mainly localized to Europe, West Africa, the Middle East, and Pakistan/West India. Lactase persistence is in fact a recent trait in human evolution, as the dominant mutation conferring persisting lactase expression appeared about 7500 years ago in Eastern Europe [22]. The high frequency of this lactase haplotype in the European

107 population indicates a strong selection pressure, which coincided with the emergence of 108 dairy cultures across Europe. Distinct mutations in the promoter region of the lactase gene 109 have been reported in West Africa and Asia, indicating that lactase persistence spread across 110 the globe through convergent evolution. The rise of lactase persistence certainly contributed 111 to the expansion of dairy farming and milk consumption in lactose-tolerant populations. The 112 increasing availability of cow's milk introduced alternatives to breast milk for young children 113 and thereby lowered the age of weaning. The resulting shortening of the nursing period also 
114 yielded a faster return to fertility in women and thus increased the birth rate in Neolithic

115 farming societies.

\section{Breast milk is a fertilizer}

117 Breast milk is the first fluid ingested by the newborn; it is the first food for the infant but it is

118 also a strong conditioner for the gut microbiota, which develops swiftly in the days following

119 birth. As documented by numerous recent studies, the gut microbiota is emerging as a

120 critical organ involved not just in intestinal physiology, but in influencing general metabolism

121 and affecting the severity of diseases such as diabetes [23] and atherosclerosis [24]. Breast

122 milk, as the product of million years of evolution, provides the optimal seeding ground for

123 the development of a healthy gut microbiota. A better understanding of the coordinated

124 action of breast milk constituents in shaping the gut microbiota will lead to a definition of

125 treatments aimed at restoring a healthy gut microbiota in diseases.

126 In addition to lactose, human breast milk comprises a large number of complex

127 oligosaccharide structures, consisting of three or more monosaccharides, which are also

128 produced in the lactating mammary epithelium. In contrast to lactose that functions as an

129 energy source to the infant, milk oligosaccharides cannot be digested by the suckling infant.

130 Human colostrum, the milk produced during the first few days after the birth of the baby,

131 contains about $22 \mathrm{~g} / \mathrm{l}$ of oligosaccharides and mature milk still about $12 \mathrm{~g} / \mathrm{l}$ [25]. Human milk

132 comprises close to 200 distinct oligosaccharides [26], a number that is by far the highest

133 among mammalian milks. This diversity is achieved by combining the four carbohydrates Gal,

134 GlcNAc, fucose (Fuc) and sialic acid (Sia) on a lactose core (Fig. 2). The term sialic acid covers

135 in fact a large family of acidic carbohydrates; $\mathrm{N}$-acetylneuraminic acid (Neu5Ac) is the only

136 sialic acid found in humans, whereas Neu5Ac and N-glycolylneuraminic acid (Neu5Gc) are

137 found in most mammals. The glycosyltransferases involved in the assembly of milk 
138 oligosaccharides are the same enzymes that build the glycans decorating glycoproteins and

139 glycolipids. The production of milk oligosaccharides is solely regulated by glycosyltransferase

140 expression in the mammary epithelium. As the program of glycosyltransferase gene

141 expression varies between mothers, the amounts of some milk oligosaccharides shows a

142 large degree of inter-individual variability [27].

143 Mammals lack the glycosidase machinery required to cleave milk oligosaccharides in the

144 gastrointestinal tract. Therefore, unaltered milk oligosaccharides reach the large intestine,

145 where they are consumed by selected bacterial taxa. The assimilation of oligosaccharides

146 requires glycosidase enzymes such as fucosidases and sialidases to break down the

147 oligosaccharides into monosaccharides, and carbohydrate transporters in order to use the

148 released monosaccharides as carbon source. Some intestinal bacteria including

149 Bifidobacterium spp. and Bacteroides spp. are well-equipped to degrade and utilize milk

150 oligosaccharides [28]. Because bacteria can have preferences for specific milk

151 oligosaccharides, differences in the composition of milk oligosaccharides impact the

152 colonization of the gut by individual bacterial groups. For example, "non-secretor" mothers,

153 who lack the fucosyltransferase FUT2, produce milk oligosaccharides devoid of $\alpha 1,2$-linked

154 Fuc. Infants of such "non-secretor" mothers show delayed intestinal colonization with

155 bifidobacteria [29], which include Fuc consumers such as Bifidobacterium

156 longum subsp. infantis and Bifidobacterium bifidum. Compositional shifts in the gut

157 microbiota induced by different milk oligosaccharide mixtures may have long-term effects

158 on the course of inflammatory diseases. For instance, elevated amounts of the milk

159 oligosaccharide Sia( $\alpha 2-3)$ lactose promote the formation of a niche for Enterobacteriaceae

160 during lactation, which extensively expands during disease and exacerbates intestinal

161 inflammation in colitis [30]. Surprisingly, Enterobacteriaceae cannot feed on Sia( $\alpha 2-3)$ lactose 
as they lack the sialidase enzymes able to cleave the capping Sia units. In fact,

163 Enterobacteriaceae rely on sialidases released by other intestinal bacteria for that task, such

164 as members of the Bacteroides genus [31]. As milk oligosaccharides are structurally similar

165 to intestinal mucin O-glycans, bacterial glycosidases also digest carbohydrates from the

166 protective mucin layer lining the intestine. The release of carbohydrates from milk

167 oligosaccharides and intestinal mucins mediated by bacterial glycosidases eventually

168 supports the "cross-feeding" of pathobionts such as Enterobacteriaceae. In the recent years

169 milk oligosaccharides and intestinal glycans have thus been recognized as key players

170 influencing the composition of the gut microbiota under healthy conditions and during

171 disease [32].

172 Maternal slgA are another component of breast milk controlling the bacterial colonization of

173 the gut. Bacterial antigens are among the epitopes recognized by maternal slgA and these

174 antibodies bind to intestinal bacteria once they reach the infant gut. Some bacterial taxa,

175 such as Enterobacteriaceae, are more widely coated than others, such as Prevotella and

176 Bacteroides [33]. Coating of bacteria with sIgA hampers their proliferation in the gut,

177 thereby preventing the expansion of colitogenic bacteria [34]. The importance of maternal

178 slgA in shaping the gut microbiota has also been demonstrated in newborn mice nursed by

179 mothers unable to transfer slgA into their milk because of a polymeric Ig receptor defect.

180 Mice fed with antibody-deficient milk presented long-lasting and detrimental changes in

181 their gut microbiota, as exemplified by increasing Pasteurellaceae and Lachnospiraceae

182 levels, and increased susceptibility towards colitis induced by dextran sulfate sodium [35].

183 The milk proteins lysozyme and lactoferrin also influence the gut microbiota by cleaving cell

184 wall polysaccharides and by chelating iron, respectively. Colostrum is especially rich in 
185

lactoferrin [36], which binds with high affinity to iron, thereby restricting its availability for the growth of pathobionts, such as Enterobacteriaceae [37].

\section{Breast milk is an umbrella}

Breast milk contains physiologically relevant amounts of bioactive proteins including immunoglobulins, cytokines, defensins and lactoferrin that contribute to the immune protection of the infant [38] (Fig. 3). Some of these immunomodulatory factors, such as macrophage colony-stimulating factor [39], are produced by epithelial cells in mammary ducts, whereas others, such as transforming growth factor $\beta$ (TGF $\beta$ ) [40], are produced by leukocytes present in the breast milk. Importantly, these bioactive proteins remain active after passage through the stomach because of a higher gastric $\mathrm{pH}$ in infants of about 3-5 compared to a gastric $\mathrm{pH}$ of 1-2 in adults. The stability of milk proteins is further maintained by $\alpha 1$-antitrypsin in early milk, which protects other proteins from gastric proteolysis [41].

The first bioactive proteins identified in the breast milk were immunoglobulins. Transfer of immunity from mother to child was described in 1903 and this effect was linked to antibodies contained in the milk. The vast majority of immunoglobulins in the breast milk belong to the IgA class. Levels of slgA reaching $12 \mathrm{~g} / \mathrm{I}$ are commonly detected in the colostrum, while mature milk contains about $1 \mathrm{~g} / \mathrm{l}$ [42]. Since the intestinal immune system at birth is immature with a low production of slgA in the first weeks of life, the high levels of slgA in the colostrum significantly contribute to the immune protection of an infant. Thus, a transfer of adaptive secretory immunity from mother to an infant in the form of slgA provides a direct protection against a variety of pathogens until the infant immune system takes over by producing sufficient slgA levels around a month after birth [43]. Lactoferrin, which reaches concentrations of $1-3 \mathrm{~g} / \mathrm{l}$ in breast milk, is another immune protective factor. Lactoferrin efficiently chelates iron, at the same time reducing the growth of certain bacteria 
relying on iron and increasing absorption of iron by the infant through binding to the

210 intestinal lactoferrin receptor ITLN1 [44]. Importantly, the cleavage of lactoferrin by pepsin

211 in the stomach yields lactoferricin, which acts as an antimicrobial peptide by disrupting the

212 membrane of gram-negative bacteria [45]. In addition, lactoferrin induces macrophage

213 phagocytosis, thereby promoting the elimination of certain bacteria [46]. The major milk

214 protein $\alpha$-lactalbumin also shares antimicrobial properties when partially unfolded and

215 associated with oleic acid [47]. The resulting complex furthermore induces apoptosis in

216 tumor cells, and has therefore been called HAMLET, for human $\alpha$-lactalbumin made lethal to

217 tumor cells. The antitumor activity of the HAMLET complex demonstrates that milk proteins

218 have therapeutic potential, as for example in treating colon cancer [48].

219 During the first weeks of life, the two anti-inflammatory cytokines interleukin-10 (IL-10) and

220 TGF $\beta$ that are transferred through breast milk contribute to the maturation of mucosal

221 immunity [38]. Indeed, the milk levels of TGF $\beta$ correlate with slgA production in breastfed

222 infants [49], and with a decreased risk for child diseases including allergy [50]. Further

223 studies performed in mice showed that milk TGF $\beta$ promotes immune tolerance to oral

224 antigens during mucosal maturation [51]. Similarly, targeted deletion of IL-10 in mice leads

225 to spontaneous enterocolitis under conventional housing conditions, which can be

226 prevented by parenteral administration of either IL-10 [52] or TGF $\beta$ [53].

227 In addition to cytokines, breast milk provides passive immune-protective factors such as

228 lysozyme, defensins [54], and soluble CD14 (sCD14) [55], which assist the infant innate

229 immune system in coping with infections (Fig. 3). The concentration of SCD14 in breast milk

230 is 20 -fold higher than in the serum of mothers. Milk $\alpha$-lactalbumin binds SCD14 and thereby

231 protects it from degradation when passing the stomach. The elevated epithelial permeability

232 in the neonate intestine enables an efficient absorption of SCD14, which sensitizes the 
233 innate immune system towards Gram-negative bacteria, thus contributing to the

234 maintenance of microbial homeostasis in the neonatal intestine.

235 Beside their prebiotic action discussed above, milk oligosaccharides also exert anti-microbial

236 functions by acting as soluble receptors for pathogens. For example, $\mathrm{H} 2$ type

237 oligosaccharides (Fig. 2) inhibit the adhesion of Campylobacter jejuni to the intestinal

238 epithelium [56], and fucosylated milk oligosaccharides from secretor mothers inhibit

239 norovirus infection [57]. Oligosaccharides carrying Lewis $X$ antigens are recognized by the

240 DC-SIGN lectin on intestinal dendritic cells. Such oligosaccharides prevent the binding of HIV

241 through DC-SIGN, thereby decreasing the presentation of the virus to CD4 ${ }^{+} \mathrm{T}_{\text {cells }}$ [58]. Some

242 milk oligosaccharides have been shown to directly regulate immune cells. For example, the

243 oligosaccharide lacto-N-fucopentaose III induces the production of IL-10 in spleen cells [59].

244 Also, oral supplementation of mice with Sia( $\alpha 2-3)$ lactose increases activation of intestinal

$245 \mathrm{CD}_{11 \mathrm{c}^{+}}$dendritic cells [60]. The activating properties of specific milk oligosaccharides may be

246 related to their structural similarity with carbohydrate epitopes found on pathogens. Indeed,

$247 \alpha 2,3$-linked Sia is present on surfaces or various pathogenic bacteria, such as group $B$

248 Streptococcus [61], Campylobacter jejuni [62], Haemophilus influenza, and Neisseria

249 meningitides [63].

250 Breast milk is a remote control

251 Besides contributing to the development of gut microbiota and the maturation of the 252 mucosal immune system, breast milk also affects metabolic pathways and supports the 253 growth of the suckling infant. Several hormones occurring in breast milk likely mediate the 254 same functions as they do as endocrine factors. Accordingly, leptin in breast milk [64] is 255 probably involved in controlling satiety and fat storage; insulin-like growth factor 1 (IGF1)

256 [65] is probably involved in stimulating body growth; and adiponectin [66] is probably 
257 involved in regulating blood glucose levels and fatty acid oxidation. Whereas these

258 hormones certainly play a role in the early growth and development of breastfed infants, the

259 true significance of milk-borne hormones is elusive as clear experimental support is difficult

260 to obtain.

261 Assessing the biological contribution of breast milk hormones is doubtless a challenging task

262 when trying to differentiate their effects from those mediated by the same hormones

263 produced endogenously. Also, the pleiotropic actions of several hormones render the

264 identification of specific effects quite difficult and can lead to ambiguous conclusions. For

265 example, some studies have attributed behavioral functions to breast milk cortisol by

266 associating cortisol levels in maternal milk with human infant temperament [67], and with

267 reduced anxiety as investigated in rats [68]. Far from discrediting such studies, it must be

268 reminded that cortisol, as the main glucocorticoid hormone, exerts multiple actions and that

269 behavioral changes may be indirect and consecutive to numerous metabolic and

270 immunologic effects. In fact, cortisol is an important factor controlling intestinal immunity

271 [69]. Accordingly, cortisol delivered through breast milk probably contributes to the

272 maintenance of anti-inflammatory conditions in the early phase of intestinal microbial

273 colonization in infants.

\section{Breast milk is a waste basket}

275 Breast milk provides various protective compounds as discussed above, but breast milk also

276 conveys lipophilic xenobiotics that accumulate in the maternal breast tissue. The list of

277 environmental contaminants is long, featuring heavy metals, pesticides, synthetic additives,

278 and endocrine disruptors among others. Thanks to vigilant scientists such as Rachel Carson

$279[70]$, who raised awareness in the general community of the health risks of such

280 contaminants, several xenobiotics have been banned over the past 20 years. For example, 
dichlorodiphenyltrichloroethane (DDT) was broadly used as pesticide in agriculture before the warning call of Rachel Carson, which eventually led to the ban of DDT in 49 countries by 1995. DDT and its metabolites affect bird reproduction and are highly toxic to fish. In humans, exposure to DDT has been associated with preterm birth [71] and increased risk for breast cancer [72]. The United States did ban the use of DDT in 1972 and several European countries already restricted the pesticide in 1970. The levels of DDT measured in the breast milk of Swedish mothers peaked by 1970 at $3 \mu \mathrm{g} / \mathrm{g}$ lipids and steadily declined to zero by the end of the twentieth century [73]. Additional studies addressing the accumulation of DDT in the human body revealed a half-life for DDT in human fatty tissues, such as breast tissue, of 290 about four years.

291 Whereas several xenobiotics have been black-listed, others are still widely used. For example, 292 phthalates are non-covalent additives found in plastics, textiles, personal-care products and 293 so forth. Phthalates are released in the environment and accumulate in fat tissues. They are 294 found in dietary products such as in butter but also in breast milk. Phthalates have been 295 claimed to act as endocrine disruptors [74]. Positive correlations have been described 296 between specific phthalates in breast milk and altered levels of sexual hormones in suckling 297 infant boys at three months of age. Especially noteworthy was the detection in such infants 298 of a higher ratio of luteinizing hormone to testosterone than is normal, which is indicative of 299 an anti-androgenic action of some phthalates [75].

300 Besides xenobiotics, breast milk is also involved in the transmission of pathogens, such as 301 HIV and cytomegalovirus, to the suckling infant. Newborns from cytomegalovirus-positive 302 mothers are protected prenatally by the transfer of anti-virus IgG through the placenta [76].

303 The situation is not as positive in the case of HIV, as transmission of the virus has been 304 documented in 10 to $40 \%$ of mother-infant pairs [77]. Consequently, the CDC recommends 
avoiding breast-feeding for HIV-positive mothers [78]. In general, only a few maternal

306 viruses are transmitted through breast milk, which underlines the general safety of

307 breastfeeding.

\section{Concluding remarks}

309 Beyond the biological functions of breast milk addressed in this review, the act of

310 breastfeeding itself is the topic of emotional discussions related to the philosophical

311 question of motherhood. Should society encourage breastfeeding simply because it is

312 "natural"? Is a woman who stops nursing her baby after three months a bad mother? Does

313 breastfeeding depreciate the economic and social status of women [79]? Similar provocative

314 questions keep the general debate on breastfeeding alive and remind us that the discussion

315 on breast milk transcends biology (see Outstanding Questions). Breast milk is ultimately why

316 Carolus Linnaeus, as the father of seven children, chose the term Mammalia to define our

317 own class of animals in the tree of life.

319 Acknowledgements

320 We thank Dr. Emma Salomonsson for reviewing the manuscript. This work was supported by

321 the Zurich Center for Integrative Human Physiology and by the Swiss National Science

322 Foundation grant CRSII3_154488/1. 


\section{Glossary}

324 Colostrum: The first milk; it is produced by the end of pregnancy and is secreted in the first

325 four days postpartum. Colostrum is rich in slgA and milk oligosaccharides, thereby providing

326 a first line of immune defense to the newborn. Bovine colostrum was used as a source of

327 anti-microbial immunoglobulins against infections before the emergence of antibiotics-

328 based therapies.

329 Defensin: Short cationic antimicrobial peptides that bind to bacterial and fungal cell walls

330 and kill microbes by destabilizing their membrane integrity. Defensins are mainly produced

331 by leukocytes and by Paneth cells in the crypts of the small intestine. Colonization of the gut

332 by microbiota stimulates the production of defensins.

333 Endocrine disruptor: Chemicals that share structural features with hormones and interfere

334 with endocrine pathways. Animals are exposed to endocrine disruptors through different

335 modes, ranging from skin contact to oral ingestion. Some endocrine disruptors are

336 environmental pollutents, such as dioxin, while others are additives to food and materials, as

337 for example bisphenol A, which is found in plastics.

338 Enterobacteriaceae: Family of Gram-negative, facultative anaerobic rod-shaped bacteria

339 encompassing Escherichia coli, Shigella, Klebsiella, Salmonella, and Yersinia. Most

340 Enterobacteriaceae reside in the intestine of animals. The Enterobacteriaceae family

341 includes commensals, pathobionts and pathogens. Enterobacteriaceae cannot process

342 oligosaccharides and large polysaccharides.

343 Mucin: Mucins are a family of highly O-glycosylated hydrophilic proteins that are the main

344 constituents of the mucus that protects epithelial layers on mucosal surfaces. Mucins are

345 also found in body fluids such as saliva and phlegm. Mucins are large proteins that are either 
346 anchored to cells through a transmembrane domain, or secreted as massive gel-like

347 aggregates. The mucin MUC2, secreted by goblet cells, is the main constituent of the thick

348 mucus layer lining the gastrointestinal tract.

349 Pathobiont: Organism that normally lives in symbiosis with a host, but can become

350 pathogenic under specific conditions such as when becoming a dominant taxa in a complex

351 environment. Typical pathobionts among gastrointestinal bacteria are Helicobacter pylori,

352 Clostridium difficile, and Escherichia coli.

353 Sialic acid: Family of 9-carbon carboxylated carbohydrates found in vertebrates and some

354 bacteria. Sialic acids are mainly found as terminal monosaccharides on glycan chains and are

355 part of carbohydrate epitopes used as receptors for viruses, such as influenza viruses, and

356 toxins, such as cholera toxin. The main forms of sialic acid found in vertebrates are N-

357 acetylneuraminic acid (NeuAc) and N-glycolylneuraminic acid (NeuGc). Humans have lost the

358 ability to synthesize NeuGc because of inactivating mutations in the $C M A H$ gene encoding

359 the cytidine monophosphate-N-acetylneuraminic acid hydroxylase enzyme.

360 Xenobiotic: A chemical compound detected in an organism that does not synthesize it.

361 Xenobiotics can mediate pharmacological and endocrine effects that can range from toxic to

362 harmless. Xenobiotics include drugs such as antibiotics and their metabolites, but also

363 environmental pollutants that accumulate through the food chain. 


\section{References}

3651 Ip, S. et al. (2007) Breastfeeding and maternal and infant health outcomes in developed 366 countries. Evid Rep Technol Assess (Full Rep), 1-186

3672 Horta, B.L. et al. (2015) Long-term consequences of breastfeeding on cholesterol, 368 obesity, systolic blood pressure and type 2 diabetes: a systematic review and meta369 analysis. Acta Paediatr 104, 30-37

3703 Chowdhury, R. et al. (2015) Breastfeeding and maternal health outcomes: a systematic 371 review and meta-analysis. Acta Paediatr 104, 96-113

3724 World Health Organization (2003) Global strategy for infant and young child feeding. 373 WHO Press, Geneva.

3745 Dewey, K.G. (1997) Energy and protein requirements during lactation. Annu Rev Nutr 17, $375 \quad 19-36$

3766 Hautvast, J.G. et al. (1989) Recommended dietary allowances for Europe. Lancet 2, 1220 3777 Prentice, A.M. et al. (1996) Energy requirements of pregnant and lactating women. Eur J $378 \quad$ Clin Nutr 50 Suppl 1, S82-110; discussion S110-111

3798 Clark, D.D. and Sokoloff, L. (1999) Circulation and Energy Metabolism of the Brain. In 380 Basic Neurochemistry: Molecular, Cellular, and Medical Aspects (6th edn) (Siegel, G.J. et 381 al., eds), pp. 637-670, Lippincott

3829 Lemay, D.G. et al. (2009) The bovine lactation genome: insights into the evolution of 383 mammalian milk. Genome Biol 10, R43

38410 Jenness, R. (1979) The composition of human milk. Semin Perinatol 3, 225-239

38511 Insull, W., Jr. et al. (1959) The fatty acids of human milk. II. Alterations produced by 386 manipulation of caloric balance and exchange of dietary fats. J Clin Invest 38, 443-450 
12 Koletzko, B. et al. (2001) Physiological aspects of human milk lipids. Early Hum Dev 65 Suppl, S3-S18

13 Weiss, G.A. et al. (2013) High levels of anti-inflammatory and pro-resolving lipid mediators lipoxins and resolvins and declining docosahexaenoic acid levels in human milk during the first month of lactation. Lipids Health Dis 12, 89

14 Strous, G.J. and Berger, E.G. (1982) Biosynthesis, intracellular transport, and release of the Golgi enzyme galactosyltransferase (lactose synthetase A protein) in HeLa cells. J Biol Chem 257, 7623-7628

15 Brodbeck, U. et al. (1967) The isolation and identification of the B protein of lactose synthetase as alpha-lactalbumin. J Biol Chem 242, 1391-1397

16 Ramakrishnan, B. and Qasba, P.K. (2001) Crystal structure of lactose synthase reveals a large conformational change in its catalytic component, the beta1,4galactosyltransferase-I. J Mol Biol 310, 205-218

17 Turkington, R.W. and Hill, R.L. (1969) Lactose synthetase: progesterone inhibition of the 401 induction of alpha-lactalbumin. Science $163,1458-1460$

18 Skovbjerg, H. et al. (1981) Purification and characterisation of amphiphilic lactase/phlorizin hydrolase from human small intestine. Eur J Biochem 114, 653-661

19 Hove, H. et al. (1999) Lactic acid bacteria and the human gastrointestinal tract. Eur J Clin Nutr 53, 339-350

40620 Taya, K. and Greenwald, G.S. (1982) Mechanisms of suppression of ovarian follicular 407 development during lactation in the rat. Biol Reprod 27, 1090-1101

40821 Brüssow, H. (2007) The quest for food : a natural history of eating. Springer

22 Coelho, M. et al. (2005) Microsatellite variation and evolution of human lactase persistence. Hum Genet 117, 329-339 
23 Wen, L. et al. (2008) Innate immunity and intestinal microbiota in the development of Type 1 diabetes. Nature 455, 1109-1113

24 Koeth, R.A. et al. (2013) Intestinal microbiota metabolism of L-carnitine, a nutrient in

$414 \quad$ red meat, promotes atherosclerosis. Nat Med 19, 576-585

41525 Kunz, C. et al. (2000) Oligosaccharides in human milk: structural, functional, and

416 metabolic aspects. Annu Rev Nutr 20, 699-722

41726 Ninonuevo, M.R. et al. (2006) A strategy for annotating the human milk glycome. J Agric $418 \quad$ Food Chem 54, 7471-7480

41927 Thurl, S. et al. (2010) Variation of human milk oligosaccharides in relation to milk groups $420 \quad$ and lactational periods. Br J Nutr 104, 1261-1271

42128 Marcobal, A. et al. (2010) Consumption of human milk oligosaccharides by gut-related 422 microbes. J Agric Food Chem 58, 5334-5340

42329 Lewis, Z.T. et al. (2015) Maternal fucosyltransferase 2 status affects the gut 424 bifidobacterial communities of breastfed infants. Microbiome 3, 13

42530 Fuhrer, A. et al. (2010) Milk sialyllactose influences colitis in mice through selective 426 intestinal bacterial colonization. J Exp Med 207, 2843-2854

42731 Huang, Y.L. et al. (2015) Sialic acid catabolism drives intestinal inflammation and 428 microbial dysbiosis in mice. Nat Commun 6, 8141. doi: 8110.1038/ncomms9141.

42932 Ng, K.M. et al. (2013) Microbiota-liberated host sugars facilitate post-antibiotic $430 \quad$ expansion of enteric pathogens. Nature $502,96-99$

43133 Tsuruta, T. et al. (2010) Development of a method for the identification of S-IgA-coated 432 bacterial composition in mouse and human feces. Biosci Biotechnol Biochem 74, 968$433 \quad 973$

43434 Palm, N.W. et al. (2014) Immunoglobulin A coating identifies colitogenic bacteria in 435 inflammatory bowel disease. Cell 158, 1000-1010 
35 Rogier, E.W. et al. (2014) Secretory antibodies in breast milk promote long-term intestinal homeostasis by regulating the gut microbiota and host gene expression. Proc Natl Acad Sci U S A 111, 3074-3079

36 Adamkin, D.H. (2012) Mother's milk, feeding strategies, and lactoferrin to prevent necrotizing enterocolitis. JPEN J Parenter Enteral Nutr 36, 25S-29S

37 Bullen, J.J. et al. (1972) Iron-binding proteins in milk and resistance to Escherichia coli infection in infants. Br Med J 1, 69-75

38 Laiho, K. et al. (2003) Breast milk fatty acids, eicosanoids, and cytokines in mothers with and without allergic disease. Pediatr Res 53, 642-647

39 Hara, T. et al. (1995) Identification of macrophage colony-stimulating factor in human milk and mammary gland epithelial cells. Pediatr Res 37, 437-443

40 Saito, S. et al. (1993) Transforming growth factor-beta (TGF-beta) in human milk. Clin Exp Immunol 94, 220-224

41 Chowanadisai, W. and Lonnerdal, B. (2002) Alpha(1)-antitrypsin and antichymotrypsin in human milk: origin, concentrations, and stability. Am J Clin Nutr 76, 828-833

42 Hanson, L.A. (1961) Comparative immunological studies of the immune globulins of human milk and of blood serum. Int Arch Allergy Appl Immunol 18, 241-267

43 Brandtzaeg, P. (2007) Induction of secretory immunity and memory at mucosal surfaces. Vaccine 25, 5467-5484

44 Suzuki, Y.A. et al. (2001) Molecular cloning and functional expression of a human intestinal lactoferrin receptor. Biochemistry 40, 15771-15779

45745 Kuwata, H. et al. (1998) Direct evidence of the generation in human stomach of an 458 antimicrobial peptide domain (lactoferricin) from ingested lactoferrin. Biochim Biophys Acta $1429,129-141$ 
460

461

462

463

464

465

466

467

468

469

470

471

472

473

474

475

476

477

478

479

480

481

482

483

46 Lima, M.F. and Kierszenbaum, F. (1987) Lactoferrin effects of phagocytic cell function. II. The presence of iron is required for the lactoferrin molecule to stimulate intracellular killing by macrophages but not to enhance the uptake of particles and microorganisms. $J$ Immunol 139, 1647-1651

47 Marks, L.R. et al. (2012) The human milk protein-lipid complex HAMLET sensitizes bacterial pathogens to traditional antimicrobial agents. PLoS One 7, e43514

48 Puthia, M. et al. (2014) Prevention and treatment of colon cancer by peroral administration of HAMLET (human alpha-lactalbumin made lethal to tumour cells). Gut

$$
63,131-142
$$

49 Ogawa, J. et al. (2004) Role of transforming growth factor-beta in breast milk for initiation of IgA production in newborn infants. Early Hum Dev 77, 67-75

50 Oddy, W.H. and Rosales, F. (2010) A systematic review of the importance of milk TGFbeta on immunological outcomes in the infant and young child. Pediatr Allergy Immunol $21,47-59$

51 Verhasselt, V. et al. (2008) Breast milk-mediated transfer of an antigen induces tolerance and protection from allergic asthma. Nat Med 14, 170-175

52 Berg, D.J. et al. (1996) Enterocolitis and colon cancer in interleukin-10-deficient mice are associated with aberrant cytokine production and CD4(+) TH1-like responses. J Clin Invest 98, 1010-1020

53 Oz, H.S. et al. (2004) Efficacy of a transforming growth factor beta 2 containing nutritional support formula in a murine model of inflammatory bowel disease. J Am Coll Nutr 23, 220-226

54 Salzman, N.H. et al. (2003) Protection against enteric salmonellosis in transgenic mice expressing a human intestinal defensin. Nature 422, 522-526 
484

485

486

487

488

489

490

491

492

493

494

495

496

497

498

499

500

501

502

503

504

505

506

507

55 Labeta, M.O. et al. (2000) Innate recognition of bacteria in human milk is mediated by a milk-derived highly expressed pattern recognition receptor, soluble CD14. J Exp Med $191,1807-1812$

56 Ruiz-Palacios, G.M. et al. (2003) Campylobacter jejuni binds intestinal $\mathrm{H}(\mathrm{O})$ antigen (Fuc alpha 1, 2Gal beta 1, 4GIcNAc), and fucosyloligosaccharides of human milk inhibit its binding and infection. J Biol Chem 278, 14112-14120

57 Jiang, X. et al. (2004) Human milk contains elements that block binding of noroviruses to human histo-blood group antigens in saliva. J Infect Dis 190, 1850-1859

58 Naarding, M.A. et al. (2005) Lewis X component in human milk binds DC-SIGN and inhibits HIV-1 transfer to CD4+ T Iymphocytes. J Clin Invest 115, 3256-3264

59 Velupillai, P. and Harn, D.A. (1994) Oligosaccharide-specific induction of interleukin 10 production by $\mathrm{B} 220+$ cells from schistosome-infected mice: a mechanism for regulation of CD4+ T-cell subsets. Proc Natl Acad Sci U S A 91, 18-22

60 Kurakevich, E. et al. (2013) Milk oligosaccharide sialyl( $(\alpha 2,3)$ lactose activates intestinal CD11c+ cells through TLR4. Proc Natl Acad Sci U S A 110, 17444-17449

61 Pritchard, D.G. et al. (1992) Murine monoclonal antibodies to type lb polysaccharide of group B streptococci bind to human milk oligosaccharides. Infect Immun 60, 1598-1602

62 Kuijf, M.L. et al. (2010) TLR4-mediated sensing of Campylobacter jejuni by dendritic cells is determined by sialylation. $J$ Immunol $185,748-755$

63 Vimr, E.R. et al. (2004) Diversity of microbial sialic acid metabolism. Microbiol Mol Biol $\operatorname{Rev} 68,132-153$

64 Quinn, E.A. et al. (2015) Maternal characteristics associated with milk leptin content in a sample of Filipino women and associations with infant weight for age. J Hum Lact 31, 273-281 
50865 Baxter, R.C. et al. (1984) Immunoreactive somatomedin-C/insulin-like growth factor I

509 and its binding protein in human milk. J Clin Endocrinol Metab 58, 955-959

51066 Martin, L.J. et al. (2006) Adiponectin is present in human milk and is associated with

511 maternal factors. Am J Clin Nutr 83, 1106-1111

51267 Grey, K.R. et al. (2013) Human milk cortisol is associated with infant temperament.

$513 \quad$ Psychoneuroendocrinology 38, 1178-1185

51468 Catalani, A. et al. (2000) Maternal corticosterone during lactation permanently affects

515 brain corticosteroid receptors, stress response and behaviour in rat progeny.

$516 \quad$ Neuroscience 100, 319-325

51769 Cima, l. et al. (2004) Intestinal epithelial cells synthesize glucocorticoids and regulate T

$518 \quad$ cell activation. J Exp Med 200, 1635-1646

51970 Carson, R. (1962) Silent spring. Fawcett Crest

52071 Longnecker, M.P. et al. (2001) Association between maternal serum concentration of

521 the DDT metabolite DDE and preterm and small-for-gestational-age babies at birth.

$522 \quad$ Lancet $358,110-114$

52372 Cohn, B.A. et al. (2015) DDT Exposure in Utero and Breast Cancer. J Clin Endocrinol

$524 \quad$ Metab 100, 2865-2872

52573 Noren, K. and Meironyte, D. (2000) Certain organochlorine and organobromine

526 contaminants in Swedish human milk in perspective of past 20-30 years. Chemosphere

$527 \quad 40,1111-1123$

52874 Albert, O. and Jegou, B. (2014) A critical assessment of the endocrine susceptibility of 529 the human testis to phthalates from fetal life to adulthood. Hum Reprod Update 20, $530 \quad 231-249$ 
53175 Main, K.M. et al. (2006) Human breast milk contamination with phthalates and

532 alterations of endogenous reproductive hormones in infants three months of age.

533 Environ Health Perspect 114, 270-276

53476 Minamishima, l. et al. (1994) Role of breast milk in acquisition of cytomegalovirus

$535 \quad$ infection. Microbiol Immunol 38, 549-552

53677 The Working Group on Mother-To-Child Transmission of HIV (1995) Rates of mother-to-

537 child transmission of HIV-1 in Africa, America, and Europe: results from 13 perinatal

538 studies. . J Acquir Immune Defic Syndr Hum Retrovirol 8, 506-510

53978 Centers for Disease Control (1985) Recommendations for assisting in the prevention of

540 perinatal transmission of human T-lymphotropic virus type III/lymphadenopathy-

541 associated virus and acquired immunodeficiency syndrome. MMWR Morb Mortal Wkly

$542 \quad \operatorname{Rep~34,~721-726,~731-722~}$

54379 Badinter, E. and Hunter, A. (2011) The conflict : how modern motherhood undermines

544 the status of women. Metropolitan Books/Henry Holt and Co. 


\section{Figure legends}

547 Figure 1. Lactose biosynthesis and degradation. Lactose synthase (PDB: 1nhe [16]) is a

548 heterodimer comprising the $\beta 1-4$ galactosyltransferase B4GALT1 and $\alpha$-lactalbumin. The

549 pituitary hormone prolactin stimulates the expression of $\alpha$-lactalbumin in the lactating

550 mammary gland. In the small intestine of the suckling infant, lactose is cleaved by lactase,

551 which expression is age-dependent. The resulting monosaccharides glucose (Glc) and

552 galactose (Gal) are absorbed by the sodium-glucose linked transporter SGLT1. In the absence

553 of lactase, lactose reaches the colon where it is degraded by intestinal microbes. The

554 increase of bacterial fermentation products causes abdominal cramps, bloating and nausea,

555 which leads to cessation of breastfeeding.

556 Figure 2. Biosynthetic pathway of milk oligosaccharides. The lactose core (boxed structure) is

557 modified by addition of fucose (Fuc), sialic acid (Sia), N-acetylglucosamine (GlcNAc) and

558 galactose (Gal). The most common breast milk trisaccharides are fucose( $\alpha 1-2)$ lactose (2FL),

559 fucose $(\alpha 1-3)$ lactose (3FL), sialyl( $\alpha 2-3)$ lactose (3SL), and sialyl( $\alpha 2-6)$ lactose (6SL). In human

560 milk, the most common tetrasaccharide is lacto-N-tetraose (LNT), whereas lacto-N-

561 neotreaose (LNnT) dominates in other mammalian milks. In human milk, Lewis antigens (LeA,

562 LeB, LeX, LeY, sLeA, sLeX) are epitopes (blue shaded structures) frequently found on milk

563 oligosaccharides. A and B blood group antigens are absent in human milk oligosaccharides,

564 but $\mathrm{O}$ antigen type I $(\mathrm{H} 1)$ and type II $(\mathrm{H} 2)$ are common. The LNT and LNnT cores can be

565 further elongated (dashed arrows) to yield oligosaccharides consisting of more than 20

566 monosaccharides.

567 Figure 3. Immune-active compounds of breast milk. Human breast milk delivers cytokines

568 (cyan circles), such as TGF $\beta$, IL-10, and M-CSF, soluble CD14 (sCD14, blue triangles),

569 lactoferrin (red circle), human milk oligosaccharides (HMO), and secreted $\operatorname{lgA}(\operatorname{sigA})$ to the 
570 infant gut. Upon resorption by the intestinal mucosa, SCD14 contributes to innate immune

571 protection by recognizing microbe-associated molecular patterns. Milk lactoferrin, slgA and

572 Paneth cell-derived defensins (orange circles) prevent bacterial (grey rods) overgrowth.

$573 \mathrm{HMO}$ act as receptor decoys, inhibiting bacterial adhesion to mucosal surfaces. HMO can be

574 also taken up by M-cells (blue cells) in Peyer's patches, which may contribute to the

575 induction of tolerogenic responses toward structurally-related mucosal glycans. In the

576 intestinal mucosa, dendritic cells (DC), macrophages (M $\Phi)$, T lymphocytes ( $T$ ), B lymphocytes

577 (B) and plasma cells (PC) orchestrate mucosal immunity through the secretion of cytokines

578 (green circles). 